\title{
Editors' Report for 2017
}

Odd-numbered years represent a time of relative stability at the editorial offices of the Journal of Economic History. William Collins is in the second year of his fouryear term as non-Eurasian editor, and Ann Carlos in her final year as senior editor and Eurasian editor. Eric Hilt continues his work as book review editor for the Americas, while Carol Shiue continues as book review editor for the rest-of-the-world. The JOURNAL is fortunate in having Lily Welch continue as the Journal Editorial Assistant and Sally Sztrecska as Production Editor.

We have benefited greatly from the expert guidance and advice of our Board of Editors and the nearly 200 referees who have contributed their time and expertise to maintaining and enhancing the quality of the JOURNAL. We are extremely grateful for and cannot overestimate their efforts. "Graduating seniors" at the Board are Hoyt Bleakley, Carlos Marichal, Chiaki Moriguchi, and Kirsten Wandschneider. We sincerely thank them for their service. The incoming board members include Latika Chaudhary, Johan Fourie, Robert Margo, John Tang, and Jessica Vechbanyongratana. Each will serve a four-year term. Numerous scholars contributed book reviews, including longer surveys, and a number of synthetic pieces. The editorial office is grateful to all who have worked on behalf of the JOURNAL over this past year.

The number of submissions to the Journal is shown in Table 1. In pulling different information from the ScholarOne system, the editorial office realized that the number of new submissions was being systematically undercounted for some years now. So, as economic historians, we show in Table 1 the previously published data and the amended data for the last seven years. Essentially, we were undercounting the number of submissions by about 20 papers in each year for the last four years. These are papers that were submitted in June of the relevant year. For details, please contact the editors. Looking at the trend, the number of submissions jumped four years ago and has been trending down. Overall, the submission rate shows a slight decline in the number of submissions. Last year saw 162 submissions to 170 in the previous year. The publication ratio displayed is the number of refereed papers and notes published in the current year divided by the number of new papers submitted in the previous year. In 2016-2017, we published 32 articles. Because of the re-counting of submissions, the publication ratio has fallen to about 0.18 but remains in line with the recent past. Table 2 reports the response-time statistics for the corresponding sample. To place the numbers into context, our goal is to have a decision back to the author within 90 days. This year's performance has been roughly on par with other years for new submissions, with a median response time of 74. The median response time for all submissions has fallen to 50 days. A small number of papers have taken much longer. We apologize to authors of pieces experiencing long delays, some of which were the result of ScholarOne system issues and a very small number of tardy reviewers. We continuously work to reduce the time for this small number of authors taking into consideration that we are, of course, concerned about the quality of the response, not just its speed.

The distributions of submissions are documented below. These data reflect the older accounting of the submissions data. They will be recalculated in next year's Editors' Notes; that said, we do not anticipate much change. We show the data for eras in Table 3 and for topics in Table 4. The automated part of the paper submission system stopped reporting on regions for a period of time, but that feature has recommenced. This, however, means that we do not have consistent comparisons over the last six years. What is currently available is shown in Table 5. 
TABLE 1

SUBMISSION AND PUBLICATION RATES

\begin{tabular}{lcccc}
\hline \hline & $\begin{array}{c}\text { Published September } \\
\text { to June issues }\end{array}$ & $\begin{array}{c}\text { New Submissions, } \\
\text { Old Method }\end{array}$ & $\begin{array}{c}\text { New Submissions, } \\
\text { New Method }\end{array}$ & $\begin{array}{c}\text { Publication } \\
\text { Ratio } \\
\text { Pt/St-1 }\end{array}$ \\
\hline $2010-2011$ & 32 & 152 & 151 & \\
$2011-2012$ & 32 & 153 & 152 & 0.21 \\
$2012-2013$ & 31 & 144 & 143 & 0.20 \\
$2013-2014$ & 32 & 159 & 158 & 0.22 \\
$2014-2015$ & 32 & 153 & 177 & 0.20 \\
$2015-2016$ & 31 & 149 & 170 & 0.18 \\
$2016-2017$ & 32 & 139 & 162 & 0.18 \\
\hline
\end{tabular}

TABLE 2

RESPONSE TIME STATISTICS

\begin{tabular}{|c|c|c|c|c|}
\hline \multirow[b]{2}{*}{ Year } & \multicolumn{2}{|c|}{ Decision Time (in days) } & \multirow[b]{2}{*}{ Mean } & \multirow[b]{2}{*}{ Median } \\
\hline & Minimum & Maximum & & \\
\hline \multicolumn{5}{|c|}{ All submissions } \\
\hline $2010 / 2011$ & 1 & 273 & 73 & 76 \\
\hline $2011 / 2012$ & 1 & 232 & 77 & 79 \\
\hline $2012 / 2013$ & 2 & 237 & 80 & 78 \\
\hline $2013 / 2014$ & 4 & 243 & 76 & 75 \\
\hline $2015 / 2015$ & 1 & 235 & 78 & 78 \\
\hline $2015 / 2016$ & 1 & 277 & 60 & 50 \\
\hline \multicolumn{5}{|l|}{ New } \\
\hline $2010 / 2011$ & 1 & 273 & 82 & 84 \\
\hline $2011 / 2012$ & 1 & 232 & 84 & 85 \\
\hline $2012 / 2013$ & 2 & 237 & 86 & 85 \\
\hline $2013 / 2014$ & 4 & 243 & 80 & 79 \\
\hline $2014 / 2015$ & 1 & 235 & 80 & 78 \\
\hline $2015 / 2016$ & 1 & 277 & 74 & 74 \\
\hline $2016 / 2017$ & 1 & 306 & 73 & 75 \\
\hline
\end{tabular}

Coverage remains broad with a continued growth in the twenty-first century as might be expected. The number of submissions on the twentieth century continues its surge passed the nineteenth century. Shown in Table 4, Growth, Political Economy, Demography and Labor are topic areas that dominated submissions.

Book reviews are an important component of the JouRnaL, and we sincerely thank the many reviewers. We also remind our membership to have their press send a copy of any new books to the relevant book review editor. The JournaL has also sought "review and reflection" synthetic articles, extended book reviews, and roundtable book reviews when appropriate. We published two Reviews and Reflections articles in Volume 77: 
TABLE 3

DISTRIBUTION OF SUBMISSIONS BY ERA, MULTIPLE SELECTIONS PER SUBMISSION POSSIBLE

\begin{tabular}{lrrrrrrr}
\hline \hline Eras & $2010-$ & $2011-$ & $2012-$ & $2013-$ & $2014-$ & $2015-$ & $2016-$ \\
\hline Pre-seventeenth century & 2011 & 2012 & 2013 & 2014 & 2015 & 2016 & 2017 \\
$\begin{array}{l}\text { Seventeenth and eighteenth } \\
\quad \text { centuries }\end{array}$ & 52 & 48 & 27 & 22 & 28 & 24 & 15 \\
Nineteenth century & 93 & 99 & 93 & 84 & 92 & 56 & 74 \\
Twentieth century & 87 & 94 & 109 & 87 & 104 & 78 & 83 \\
Twenty-first century & 11 & 11 & 15 & 16 & 12 & 15 & 16 \\
Not applicable & 6 & 6 & 2 & 2 & 2 & 3 & 4 \\
$\quad$ Total & 273 & 284 & 286 & 250 & 281 & 200 & 219 \\
\hline & & & & Percent & & & \\
\hline Pre-seventeenth century & 8.8 & 9.2 & 9.4 & 8.8 & 10.0 & 12.0 & 6.8 \\
Seventeenth and eighteenth & 19.0 & 16.9 & 14.0 & 15.6 & 15.3 & 12.0 & 12.3 \\
$\quad$ centuries & & & & & & & \\
Nineteenth century & 34.1 & 34.9 & 32.5 & 33.6 & 32.7 & 28.0 & 33.8 \\
Twentieth century & 31.9 & 33.1 & 38.1 & 34.8 & 37.0 & 39.0 & 37.9 \\
Twenty-first century & 4.0 & 3.9 & 5.2 & 6.4 & 4.3 & 7.5 & 7.3 \\
Not applicable & 2.2 & 2.1 & 0.7 & 0.8 & 0.7 & 1.5 & 1.8 \\
\hline
\end{tabular}

"Plague and Lethal Epidemics in the Pre-Industrial World" by Guido Alfani and Tommy Murphy in the March 2017 issue and "Economic History, Historical Analysis, and the 'New History of Capitalism" " by Eric Hilt in the June 2017 issue.

Readers will recall that at the 2013 meetings, the EHA board of trustees approved a policy to make publication conditional on archiving the data sufficient to replicate the results in the accepted articles. The new rules read:

"It is the policy of the Journal of Economic History to publish papers only if the data used in the analysis are clearly and precisely documented and are readily available to any researcher for purposes of replication. Authors of accepted papers must archive, prior to publication, the data, programs, and other details of the computations sufficient to permit replication. These will be archived at ICPSR and a stable URL link to these data will published with each article. The Editors should be notified at the time of submission if the data used in a paper are proprietary or if, for some other reason, the requirements above cannot be met.

As soon as possible after acceptance, authors are expected to deposit their data, programs, and sufficient details to permit replication with ICPSR. Questions regarding any aspect of this policy should be forwarded to the Journal Editor." 
TABLE 4

DISTRIBUTION OF SUBMISSIONS BY TOPIC, MULTIPLE SELECTIONS PER SUBMISSION POSSIBLE

\begin{tabular}{|c|c|c|c|c|c|c|}
\hline Topics & $\begin{array}{c}2011- \\
2012\end{array}$ & $\begin{array}{c}2012- \\
2013\end{array}$ & $\begin{array}{c}2013- \\
2014\end{array}$ & $\begin{array}{c}2014- \\
2015\end{array}$ & $\begin{array}{c}2015- \\
2016\end{array}$ & $\begin{array}{c}2016- \\
2017\end{array}$ \\
\hline Agriculture & 16 & 18 & 24 & 19 & 8 & 10 \\
\hline Demography & 13 & 18 & 25 & 24 & 25 & 22 \\
\hline Growth & 39 & 40 & 26 & 39 & 36 & 28 \\
\hline History of thought & 2 & 5 & 3 & 4 & 7 & 3 \\
\hline Industry & 19 & 19 & 20 & 19 & 8 & 7 \\
\hline International trade, finance & 24 & 30 & 31 & 20 & 15 & 20 \\
\hline Labor & 26 & 25 & 22 & 23 & 24 & 31 \\
\hline Money and macro & 23 & 21 & 17 & 23 & 16 & 11 \\
\hline Other & 23 & 11 & 22 & 18 & 10 & 12 \\
\hline Political economy & 45 & 42 & 36 & 35 & 31 & 41 \\
\hline Private finance, capital markets & 28 & 23 & 19 & 19 & 12 & 12 \\
\hline Public finance & 16 & 14 & 17 & 17 & 12 & 9 \\
\hline Technology & 13 & 10 & 9 & 13 & 11 & 11 \\
\hline Urban and regional & 9 & 14 & 10 & 10 & 13 & 13 \\
\hline \multirow[t]{2}{*}{ Total } & 296 & 290 & 281 & 291 & 228 & 230 \\
\hline & \multicolumn{6}{|c|}{ Percent } \\
\hline Agriculture & 5.4 & 6.2 & 8.5 & 6.7 & 3.5 & 4.3 \\
\hline Demography & 4.4 & 6.2 & 8.9 & 8.2 & 11.0 & 9.6 \\
\hline Growth & 13.2 & 13.8 & 9.3 & 13.8 & 15.8 & 12.2 \\
\hline History of thought & 0.7 & 1.7 & 1.1 & 1.3 & 3.0 & 1.3 \\
\hline Industry & 6.4 & 6.6 & 7.1 & 6.7 & 3.5 & 3.0 \\
\hline International trade, finance & 8.1 & 10.3 & 11.0 & 10.0 & 6.6 & 8.7 \\
\hline Labor & 8.8 & 8.6 & 7.8 & 8.0 & 10.5 & 13.5 \\
\hline Money and macro & 7.8 & 7.2 & 6.0 & 8.0 & 7.0 & 4.8 \\
\hline Other & 7.8 & 3.8 & 7.8 & 6.2 & 4.4 & 5.2 \\
\hline Political economy & 15.2 & 14.5 & 12.8 & 12.0 & 13.6 & 17.8 \\
\hline Private finance, capital markets & 9.5 & 7.9 & 6.8 & 6.5 & 5.2 & 5.2 \\
\hline Public finance & 5.4 & 4.8 & 6.0 & 5.8 & 5.2 & 3.9 \\
\hline Technology & 4.4 & 3.4 & 3.2 & 4.1 & 4.8 & 4.8 \\
\hline Urban and regional & 3.0 & 4.8 & 3.6 & 3.5 & 5.7 & 5.7 \\
\hline
\end{tabular}

All new submissions from 1 January 2016 required authors to post data and replication files to maintain and advance the JouRnAL's efforts to ensure transparency and scholarly integrity. A few papers submitted prior to this date are still in process and those authors have been strongly encouraged to follow the new policy. A branded page can be found with openICPSR and depositors can submit files into openICPSR per the instructions available at: http://www.icpsr.umich.edu/files/openICPSR/JEH-depositinstructions.pdf.

The editorial office and ICPSR continue to work to enhance the interface. 
TABLE 5

DISTRIBUTION OF GEOGRAPHICAL AREA

\begin{tabular}{|c|c|c|c|c|c|c|c|}
\hline Regions & $\begin{array}{c}2010- \\
2011\end{array}$ & $\begin{array}{l}2011- \\
2012\end{array}$ & $\begin{array}{c}2012- \\
2013\end{array}$ & $\begin{array}{c}2013- \\
2014\end{array}$ & $\begin{array}{c}* 2014- \\
2015\end{array}$ & $\begin{array}{l}* * 2015- \\
2016\end{array}$ & $\begin{array}{l}2016 \\
2017\end{array}$ \\
\hline Africa & 7 & 7 & 8 & 8 & 3 & 2 & 13 \\
\hline Asia & 21 & 22 & 38 & 19 & 4 & 6 & 28 \\
\hline Australia, New Zealand & 1 & 3 & 8 & 3 & 3 & 0 & 5 \\
\hline Eastern Europe/Russia & 14 & 9 & 12 & 15 & 2 & 3 & 13 \\
\hline Great Britain & 44 & 34 & 27 & 36 & 10 & 2 & 29 \\
\hline Latin America & 11 & 20 & 15 & 19 & 4 & 1 & 6 \\
\hline Middle East & 9 & 7 & 12 & 7 & 2 & 3 & 9 \\
\hline $\begin{array}{l}\text { Non-Spanish speaking } \\
\text { Caribbean }\end{array}$ & 1 & 1 & 0 & 4 & 0 & 1 & 1 \\
\hline United States and Canada & 76 & 68 & 65 & 65 & 16 & 10 & 48 \\
\hline Western Europe & 64 & 77 & 65 & 56 & 14 & 6 & 49 \\
\hline Not applicable & 7 & 10 & 9 & 3 & 0 & 3 & 2 \\
\hline \multirow[t]{2}{*}{ Total } & 255 & 258 & 259 & 235 & 58 & 37 & 203 \\
\hline & \multicolumn{7}{|c|}{ Percent } \\
\hline Africa & 2.7 & 2.7 & 3.1 & 3.4 & 5.2 & 5.4 & 6.4 \\
\hline Asia & 8.2 & 8.5 & 14.7 & 8.1 & 6.9 & 16.0 & 13.8 \\
\hline Australia and New Zealand & 0.4 & 1.2 & 3.1 & 1.3 & 5.2 & 0.0 & 2.5 \\
\hline Eastern Europe/Russia & 5.5 & 3.5 & 4.6 & 6.4 & 3.4 & 8.1 & 6.4 \\
\hline Great Britain & 17.3 & 13.2 & 10.4 & 15.3 & 17.2 & 5.4 & 14.3 \\
\hline Latin America & 4.3 & 7.8 & 5.8 & 8.1 & 6.9 & 2.7 & 3.0 \\
\hline Middle East & 3.5 & 2.7 & 4.6 & 3.0 & 3.4 & 8.1 & 4.4 \\
\hline $\begin{array}{l}\text { Non-Spanish speaking } \\
\text { Caribbean }\end{array}$ & 0.4 & 0.4 & 0.0 & 1.7 & 0.0 & 2.7 & 0.5 \\
\hline United States and Canada & 29.8 & 26.4 & 25.1 & 27.7 & 27.6 & 27.0 & 23.6 \\
\hline Western Europe & 25.1 & 29.8 & 25.1 & 23.8 & 24.1 & 16.2 & 24.1 \\
\hline Not applicable & 2.7 & 3.9 & 3.5 & 1.3 & 0.0 & 8.1 & 1.0 \\
\hline
\end{tabular}

*2014-2015 Region data are from 1 July 2014-14 October 2015.

**2015-2016 Region data are from 18 March 2016-30 June 2016.

Referees for 2016 were:

Olivier Accominotti

Philipp Ager

Gani Aldashev

Sylvia Allegretto

Bob Allen

Carlos Alvarez Nogal

Pamfili Antipa

Leticia Arroyo Abad
Cihan Artunç

Ragui Assaad

Gareth Austin

Rudiger Bachmann

Martha Bailey

Andy Baker

Richard Baker

Gerben Bakker
Victoria Bateman

Joerg Baten

Brian Beach

Jessica Bean

Asaf Berstein

Hoyt Bleakley

Matthias Blum

Gustavo Bobonis 
Howard Bodenhorn

Lars Boerner

Karol Borowiecki

Maristella Botticini

Leah Boustan

H. V. Bowen

George Boyer

Fabio Braggion

Stephen Broadberry

John Brown

Liam Brunt

Justin Bucciferro

Carsten Burhop

Charles Calomiris

Gareth Campbell

Forrest Capie

Leonard Carlson

Celeste Carruthers

Youssef Cassis

Benjamin Chabot

David Chambers

Eric Chaney

Latika Chaudhary

Gregory Clark

Karen Clay

David Clingingsmith

Patrick Coe

Lisa Cook

Lee A. Craig

Neil Cummins

Ludo Cuyvers

Tomas Cvrcek

Aditya Dasgupta

Alan de Bromhead

Melissa Dell

Catherine Desbarats

Livio Di Matteo

Mark Dincecco

Jordi Domenech

Fabian Drixler

Nicolas Duquette

Alan Dye

Zvi Eckstein

Marc Egnal

Tamer el-Leithy

Shari Eli

Jari Eloranta
Ruben Enikolopov

Steven A. Epstein

Katherine Eriksson

Jose-Antonio Espin-Sanchez

Thibault Fally

David Feeny

James Feigenbaum

James Fenske

Alan Fernihough

Daniel Fetter

Price Fishback

Jonathan Fisher

Caroline Fohlin

Mauricio Font

Johan Fourie

Ewout Frankema

Carola Frydman

Dustin Frye

David Galenson

Kenneth Garbade

Leigh Gardner

Samuel Garrido

R. Richard Geddes

James Gerber

Michela Giorcelli

William N. Goetzman

Rafael Gonzalez-Val

Kathryn Graddy

Regina Grafe

Amanda Gregg

Paul Gregory

Avner Greif

Ben Groom

Pauline Grosjean

Richard Grossman

Catherine Guirkinger

Federico Gutierrez

Michael Haines

Gillian Hamilton

Christopher Hanes

Leslie Hannah

C. Knick Harley

Timothy Hatton

Philipp Hauber

Joshua Hausman

Scott Hemphill

Carlos Eduardo Hernandez
Henning Hillmann

Richard Hornbeck

Erik Hornung

Elise Huillery

Robert Inklaar

Kris Inwood

Alejandra Irigoin

Douglas Irwin

Denis Ivanov

David Jacks

Andrew Jalil

Matthew Jaremski

Taylor Jaworski

Morten Jerven

Joost Jonker

Gisella Kagy

Morgan Kelly

Lionel Kesztenbaum

Zorina Khan

Sukkoo Kim

Christopher Kingston

Carl Kitchens

Edward Kosack

Sumner La Croix

Ryan Lampe

Tim Larsen

Tomas Larsson

Changkeun Lee

Sibylle Lehmann

Adrian Leonard

Margaret Levenstein

Byron Lew

Frank Lewis

Joshua Lewis

Dan Li

Amy Liu

Jonas Ljungberg

Jason Long

Dean Lueck

Seth Mallios

Robert Margo

James Markusen

Catherine Massey

Anne E. C. McCants

John J. McCusker

Terra McKinnish

Christopher Meissner 
Guy Michaels

Grant Miller

Melinda Miller

David Mitch

Carolyn Moehling

Jon Moen

Alexander Moradi

Petra Moser

Carl Mosk

Jose Moya

Tomas Murphy

Aldo Musacchio

Suresh Naidu

Joana Naritomi

Larry Neal

Todd Neumann

Dennis Novy

Nathan Nunn

Cormac Ó Gráda

Trevor O'Grady

Donal O'Neill

Kevin O'Rourke

Mary O'Sullivan

Sheilagh Ogilvie

Claudia Olivetti

Alan L. Olmstead

Kim Oosterlinck

Alexander Opitz

Sonja Opper

Craig Palsson

Sevket Pamuk

John Parman

Elisabeth Perlman

Alexander Persaud

Leandro Prados de la Escosura

Nishith Prakash

Stephen Quinn

Roger Ransom

Sarah Reber

Scott Redenius

Riad Rezzik

Paul Rhode

Gary Richardson

Evan Roberts

Hugh Rockoff

Jonathan Rose

Elyce J. Rotella
Peter Rousseau

Jared Rubin

Mohamed Saleh

Laura Salisbury

Kristina Sargent

Walter Scheidel

Max-Stephan Schulze

John Sedgwick

Edson Severnini

Gary S. Shea

Allison Shertzer

Katharine Shester

Carol Shiue

Richard Sicotte

Petra Sijpesteijn

James Simpson

David P. Sims

Michael Sinkinson

Steven Smith

Kenneth A. Snowden

Yannay Spitzer

Steven Sprick Schuster

Richard Steckel

William Summerhill

Richard Sutch

Patrick Svensson

John Tang

Peter Temin

Sharon Tennyson

Michèle Tertilt

Jose Tessada

Werner Troesken

John Turner

Andrey Ukhov

Bas van Leeuwen

Marlous van Waijenburg

Jan Luiten Van Zanden

Jordi Vidal-Robert

Jacob Vigdor

Carlos Villarreal

Nico Voigtländer

Tamas Vonyo

Ilya Voskoboynikov

Daniel Waldenstrom

John Wallis

Patrick Wallis

Lorena Walsh
Randall Walsh

Marianne Wanamaker

Kirsten Wandschneider

Zachary Ward

Warren Weber

Marc Weidenmier

Bruce Weinberg

David C. Wheelock

Eugene White

Michelle White

Glenn Withers

Nikolaus Wolf

Gavin Wright

Robert Wright

Yiqing Xie

Guo Xu

Se Yan

Noam Yuchtman

Daniel Ziblatt

Nicolas Ziebarth

Ariell Zimran 